\title{
DPS-AA: Intranet Migration Strategy Model for Clouds
}

\author{
Abebe Alambo Tona ${ }^{1}$ \\ Lecturer, Gambella University, Department of Computer Science, Gambella, 126, Ethiopia \\ Email: abebealambo@gmail.com
}

Durga Prasad Sharma ${ }^{2}$

Professor \& Adviser, AMUiT, MOEFDRE under UNDP \& MAISM-RTU Kota

Email:dp.shiv08@gmail.com

Received: 21 December 2019; Accepted: 19 February 2020; Published: 08 October 2020

\begin{abstract}
Intranets \& Intranet-wares have become a central junction/platform for implementing the organization's specific work culture like computing, communication and collaboration. In order to ensure smooth and effective communication, computing and collaboration among employees, intranets play an important role. For vertical and horizontal management, we use intranet-wares for directing, reporting, collaborating, socializing, communicating and meeting or discussing the professional and social issues. Today, cloud-based computing, communication, and collaboration have created new frontiers and emerging paradigms towards re-engineering of work cultures in the organizations. In order to enhance the performance with extended features, next-generation computing, communication, and collaboration the intranet needs redesign and migration strategy over alternative technology platforms. This research paper tries to answer the research questions that how an alternative technology strategy or pathway can be explored for enhancing the performance and extending the features of the exiting designs of educational Intranets. Further; how an on-premise intranet can be migrated over cloud platforms with enhanced performance and extended/add-on features. After analysis of collected facts, understanding the issues, challenges and limitations of the existing state of art intranets, a strong need for performance enhancement and add on features was observed for Intranets. The study deeply investigated and analyzed the issues, challenges and limitations i.e. features and performances of the current state of the art of the intranets in general and on-premise Intranet of AMU in specific. Finally, an Intranet Migration Strategy Model over Hybrid Cloud was designed and developed using SaaS (i.e. AMU CloudNet). In this study, the Interact Intranet was used for designing and demonstrating the functional prototype of intranet show that how computing, communication and collaboration services can be enhanced with anytime, anywhere and boundary-less access.
\end{abstract}

Index Terms: Intranet, Intranet-wares, computing, communication, Migration Strategy Model, Cloud, On-premise

\section{Introduction}

An intranet is a set of networks, which uses Internet Protocol, and IP-based tools such as web browsers and file transfer applications under the control of a single administrative entity. The most commonly, an Intranet is the internal network of an organization, which typically has at least one web server to provide users with organizational information. It is a network designed for distributing the information resources to each individual within the organization domain using TCP/IP or may be other protocol-based technologies. It is commonly used in different types of organizations and has many similarities to an Internet, but the difference is that an Intranet is local, while the Internet is global and in other words, an Intranet (a secure information-sharing system) uses data stored on an internal corporate network, while the internet uses data stored on the servers all around the world. The Intranet is privately owned by an organization however, Internet is not owned by a single organization. By its definition, Internet is a worldwide network of networks. The purpose of an Intranet differs according to the type of organization where it is implemented [1, 2, 3, 4, 5].

A. Why Intranet?

Although, the concept of Intranet depends heavily on the internet and related technology, the need for Intranet arises more from the academic environment to transform the way how official activities are performed. The points listed below are some of the important motivational features of Intranet that makes it killer application environment. These features are Project Management [1], Variability in the Working Environment, and Rapid Change in Academic 
Environment, Support to Stakeholders, Employee Collaboration, Socialization, and Cost-Cuts. Thus, Intranet provides access to corporate information repository to the authorized user with a minimal cost, time and effort $[5,6]$.

This research study is an effort to design a migration strategy model for next generation intranets having ondemand Scalability, Open boundary Accessibility, Service reliability, High Availability (Uptime), High responsive, Fault tolerant with promised Security, Intelligent Search Content Filtration, Cost-effectiveness, and Anytime, Anywhere over Any device Accessibility. This design is proposed to be Migration Strategy Model for existing intranet designs. The major aim to improve the performance and add on advanced features [1].

\section{B. Cloud Computing}

Cloud computing is the next generation technology. Cloud is the next natural step in the evolution of on-demand information technology services and products. Actually, the Cloud is a metaphor for the Internet, based on how it is depicted in computer network diagrams, and is an abstraction for the complex infrastructure it conceals. It is a style of computing in which IT-related capabilities are provided "as a service", allowing users to access technology-enabled services via the Internet (i.e. the Cloud) without knowledge of expertise or control over the technology infrastructure that supports them [1].

Today, the computing industry is migrating towards service-based models like Platform as a Service (PaaS), Infrastructure as a Service (IaaS) and Software as a Service (SaaS) for consumers and enterprises to access on-demand services / products regardless of time and location. There will be an incredible increase in the number of Cloud platforms available today. Cloud computing is a new paradigm in the fields of technology and system migration that focuses on sharing data and computational resources via or over a scalable worldwide network of nodes (such as end user's computer, data centers, and web services). Therefore, this network of nodes is virtualized as a cloud. As a primary observation, "haves and have not" are serious issues in on-premise Intranet vs. Intranet over Cloud. [2, 3, 5, 7].

\section{Intranet over Cloud}

Intranet over cloud is proposed to be the next generation technology strategy for boundary-less access at any time for the user's community via internet. The Cloud based Intranet Migration Model especially designed for the academicians, researchers and students can enhance the systems efficiency and performance in compare to the traditional/on-premise Intranets. The next generation intranets needs on-demand Scalability, Open boundary Accessibility, Service reliability, High Availability (Uptime), Cost effectiveness, High responsive, fault tolerant and high Performance with promised Security, Anytime, Anywhere over Any device Availability[4,5,7,8]. As on date such issues, challenges and limitations (on-demand Scalability, Open boundary Accessibility, Service reliability, High Availability (Uptime), High responsive, Fault tolerant with promised Security, Intelligent Search Content Filtration, Cost-effectiveness, and Anytime, Anywhere over Any device Accessibility) in the existing designs and practices over intranets are not addressed by specific design or models. This makes this research a worth initiating activity towards a new knowledge contribution in the domain and the same has been proved and mentioned in the table 1 under the review of literature section.

\section{Review of Literature}

In this research paper, many researchers' works are critically reviewed. The following points in the table-1 are presented about the related research works with significant contributions and critical remarks.

Table 1. Summary of Focused and Related Research Works in Reference to this Study

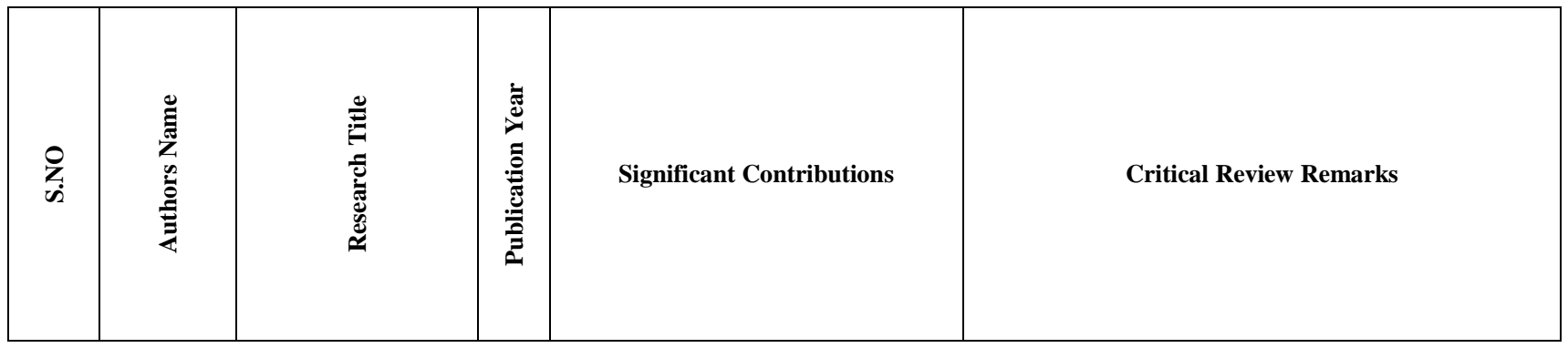




\begin{tabular}{|c|c|c|c|c|c|}
\hline 1 & 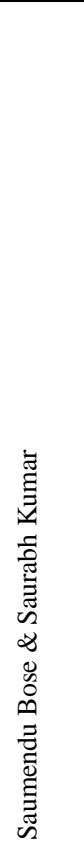 & 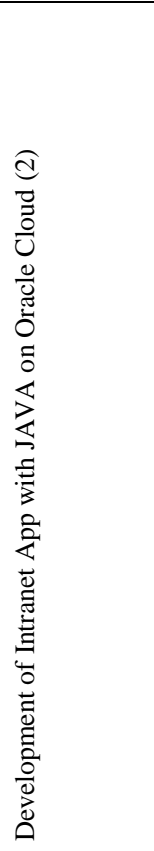 & $\stackrel{\Delta}{\stackrel{d}{\sigma}}$ & $\begin{array}{l}\text { Developing the applications \& } \\
\text { deploying it on local servers, rather } \\
\text { than on users' desktops to reduce } \\
\text { costs of deployment and } \\
\text { maintenance, as well as reducing } \\
\text { power consumption. }\end{array}$ & $\begin{array}{l}\text { Relevant to this study but, Intranet is maintained over } \\
\text { the local or on premise servers. In proposed study, } \\
\text { Intranet is proposed to be maintained over the global } \\
\text { cloud for overcoming the limitations of existing } \\
\text { intranets like on-demand Scalability, Open boundary } \\
\text { Accessibility, Service reliability, High Availability } \\
\text { (Uptime), High responsive, Fault tolerant with } \\
\text { promised Security, Intelligent Search Content } \\
\text { Filtration, Cost-effectiveness, and Anytime, } \\
\text { Anywhere over Any device Accessibility. }\end{array}$ \\
\hline 2 & 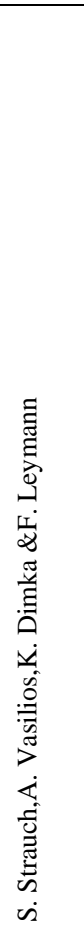 & 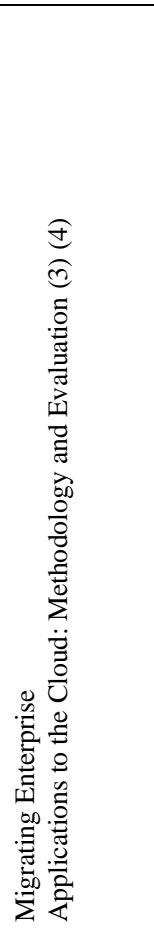 & $\stackrel{ \pm}{\vec{N}}$ & $\begin{array}{l}\text { Migrating an enterprise application } \\
\text { over the cloud platform using } \\
\text { application refactoring method to } \\
\text { assist application developers }\end{array}$ & $\begin{array}{l}\text { Relevant to this study but, in proposed study not only } \\
\text { application developers are assisted i.e. all Stakeholders } \\
\text { of the organization are proposed to be benefited from } \\
\text { usage point of views. The drawback of this study was a } \\
\text { lack of focus on the points; on-demand Scalability, } \\
\text { Open boundary Accessibility, Service reliability, High } \\
\text { Availability (Uptime), High responsive, Fault tolerant } \\
\text { with promised Security, Intelligent Search Content } \\
\text { Filtration, Cost-effectiveness, and Anytime, } \\
\text { Anywhere over Any device Accessibility. }\end{array}$ \\
\hline 3 & 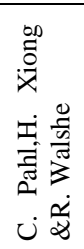 & 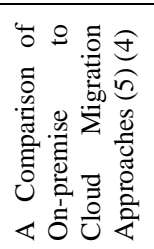 & $\frac{m}{\stackrel{\sim}{n}}$ & $\begin{array}{l}\text { Providing an overview of different } \\
\text { types of cloud computing } \\
\text { deployment models and describes } \\
\text { that which one to opt so that the } \\
\text { security requirements and goals of } \\
\text { the organization can met }\end{array}$ & $\begin{array}{l}\text { Relevant to this study because it discusses about the } \\
\text { selection of the best fit deployment models for the } \\
\text { organizations computing platforms. In this study } \\
\text { intranet over cloud requires clear cut decision about } \\
\text { deployment model but other target issues and } \\
\text { challenges are again overlooked }\end{array}$ \\
\hline
\end{tabular}




\begin{tabular}{|c|c|c|c|c|c|}
\hline 4 & 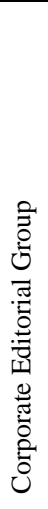 & 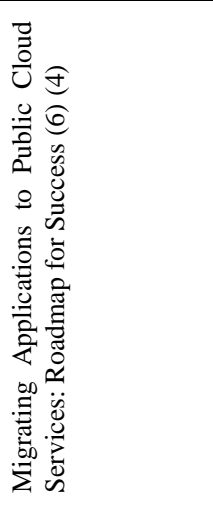 & $\stackrel{m}{\vec{D}}$ & $\begin{array}{l}\text { Shows Strategic \& methodical } \\
\text { consideration to the organization for } \\
\text { thoroughly assessing and } \\
\text { determining that which application } \\
\text { workloads can benefit most from } \\
\text { early migration to the cloud } \\
\text { migration }\end{array}$ & $\begin{array}{l}\text { Mostly relevant to the proposed study. The } \\
\text { identification and classification of application } \\
\text { workloads running on the existing platform is } \\
\text { considered before migration over the cloud especially } \\
\text { in reference to intranet, it is desirable. As per our view } \\
\text { the features like on-demand Scalability, Open } \\
\text { boundary Accessibility, Service reliability, High } \\
\text { Availability (Uptime), High responsive, Fault tolerant } \\
\text { with promised Security, Intelligent Search Content } \\
\text { Filtration, Cost-effectiveness. Anytime, Anywhere } \\
\text { over Any device Accessibility, and are not considered } \\
\text { specifically to understand the best benefits and features } \\
\text { of the intranet. }\end{array}$ \\
\hline in & 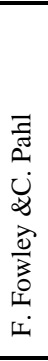 & 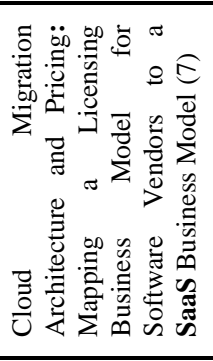 & 号 & $\begin{array}{l}\text { Investigated cloud architecture \& } \\
\text { software utilization costing with } \\
\text { business models before migrating an } \\
\text { in-house developed intranet. It } \\
\text { provided product onto a cloud IaaS } \\
\text { or PaaS platform with mapping a } \\
\text { licensing model onto a cloud } \\
\text { monetization model along with } \\
\text { income \& expenses in the cloud in } \\
\text { relation to the chosen cloud } \\
\text { delivery model }\end{array}$ & $\begin{array}{l}\text { Relevant to this study because investigation \& analysis } \\
\text { of the existing system with reference to costing is } \\
\text { required in the proposed study for intranet migration as } \\
\text { well. Except the cost other features like on-demand } \\
\text { Scalability, Open boundary Accessibility, Service } \\
\text { reliability, High Availability (Uptime), High } \\
\text { responsive, Fault tolerant with promised Security, } \\
\text { Intelligent Search Content Filtration, and Anytime, } \\
\text { Anywhere over Any device Accessibility. are again } \\
\text { overlooked }\end{array}$ \\
\hline 6 & 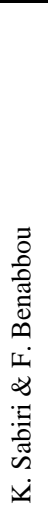 & 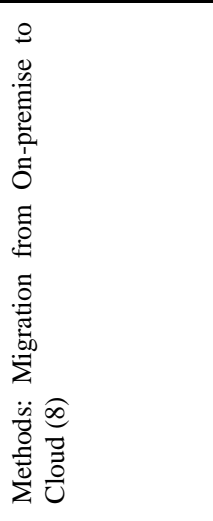 & $\frac{n}{\stackrel{d}{c}}$ & $\begin{array}{l}\text { This study is identifying \& analyzing } \\
\text { different types of cloud service } \\
\text { migration methods \& strategies } \\
\text { before Migrating a legacy style } \\
\text { applications to the cloud computing } \\
\text { environment }\end{array}$ & $\begin{array}{l}\text { Relevant to this study, as the selection of service } \\
\text { delivery models (like IaaS, PaaS, and SaaS) and } \\
\text { analyzing their Migration strategy is the most } \\
\text { important part before deciding to move to the cloud. } \\
\text { Throughout the study the aforementioned features are } \\
\text { still missing and it makes the proposed study very } \\
\text { relevant and worth doing for new knowledge } \\
\text { contribution. }\end{array}$ \\
\hline
\end{tabular}

\section{Methodology}

\section{A. Research Design}

This study by nature is the mixed version of exploratory and constructive research. Initially this research starts with an effort to explore the possibility of Migrating intranet over cloud for performance and feature enhancements. It explores the answer of the several questions for migrating intranet over cloud with new knowledge in terms of better performance and add-on features. Further, the research constructs a migration strategy model with functional prototype designed over Interact-Intranet platform. Hence, this is exploratory constructive research design. The primary data collection procedure is presented in fig. 1 for understanding and analyzing the existing facts about issues, challenges and limitations of current state of art practices and features of existing intranets.

\section{B. Research Approach}

In order to achieve the general and specific objectives alongwith answer of the research questions, this study used different approaches/methods for gathering relevant data in the area of research. Fig. 1 presents the major data gathering approaches used in this study. The Research Approach used for this research study is Mixed (Quantitative for user's community and Qualitative for professionals and experts and Personal Technical Observation by the researchers self).

\section{Data Gathering}

In this research study, data gathering techniques used survey with structured close ended questionnaire for end users like instructor/academicians and technicians, interview with open ended questionnaire for expert professionals like ICT Director, selected University higher officials like Vice Presidents, Directors and data/network admin were used. 


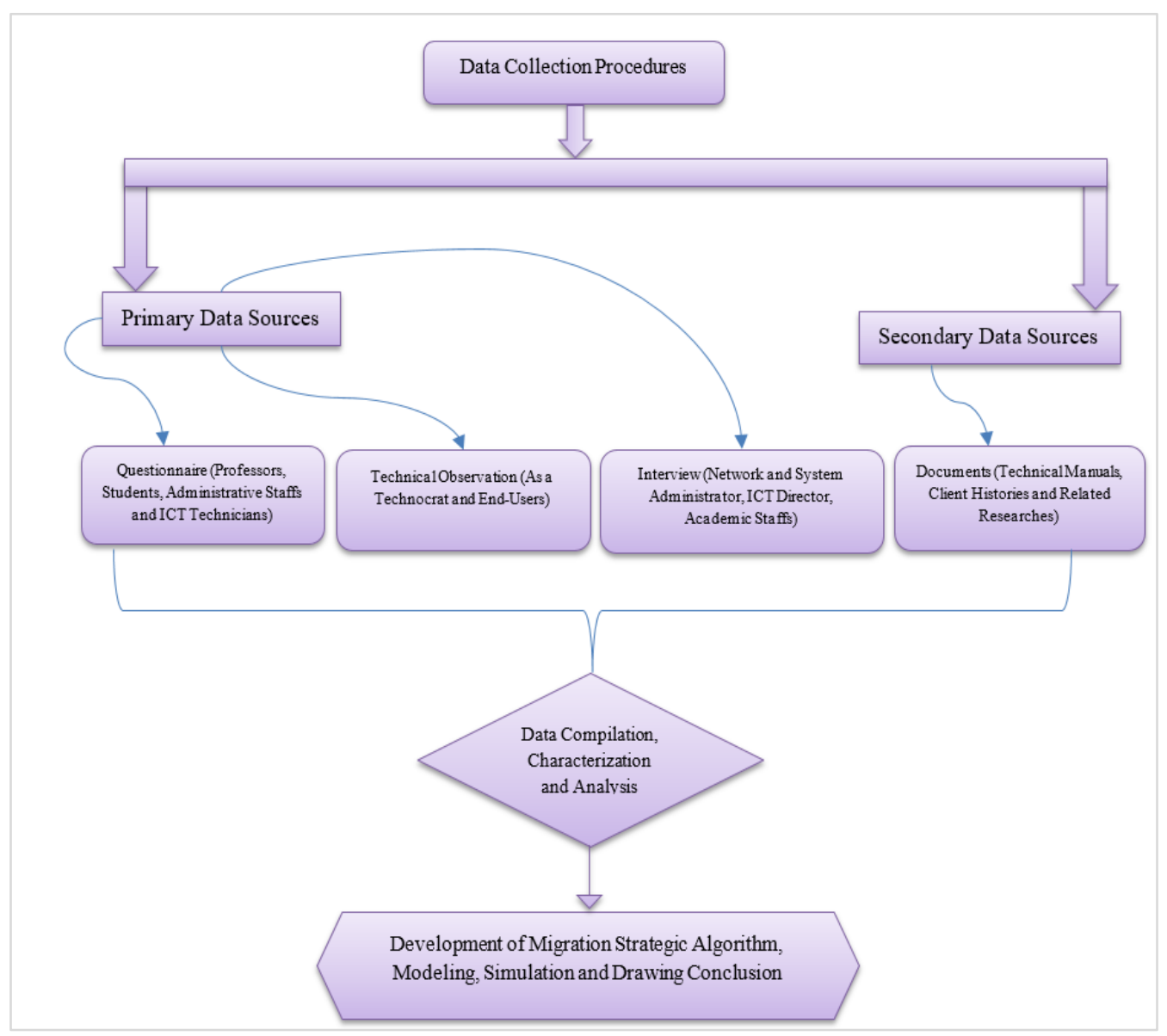

Fig. 1. Data Collection Procedures

The end user's awareness towards alternative Intranet model like Intranet over cloud vs., on-premise existing intranets for on-demand Scalability, Open boundary Accessibility, Service reliability, High Availability (Uptime), High responsive, Fault tolerant with promised Security, Intelligent Search Content Filtration, Cost-effectiveness, and Anytime, Anywhere over Any device Accessibility features data were collected and analyzed. In this section other robust features promised by alternative models were also analyzed for Smart and Intelligent Search for filtered contents.

This study focuses on intranets in general and university intranets in specific. As a case analysis, this study selected the Intranet of Arba Minch University (AMU) for assessing its standardized performance and features. This intranet provides many ICT enabled services efficiently and effectively. However, in current digital revolutions; if a system completes its five years of age, it needs to be reviewed or reorganized so as update and upgrade in the light of modernized systems and technologies towards better sustainability and performance. If it continued in its old ways, it can go beyond the control, governance and management and the same is true about the existing state of art Intranets. In order to align the existing state of art intranet models with the technological advancements, an open-minded scientific review and careful re-planning is required.

The developing countries like Ethiopia have organizations where studies and analysis for smart technology adoption [8] and adaptions are lagging behind. The encouraging support for cloud computing adoption in business, education and other fields are required to be improved.

Based on the survey, interview inputs and technical observations of researcher, it has been observed and analyzed that the awareness of user's community towards modernized Intranets like Intranet over cloud is missing in planning, designing and developments. Using new features like horizontal and vertical scalability, salable capacity, cross boundary accessibility, high uptime, information sharing dynamism, etc. has not been plasticized over existing state of art intranets in educational institutions of Ethiopia.

\section{Prototype Designing}

In this research study, the Hybrid Cloud [9, 24] was proposed for deployment of the services of Intranet as the university functions consists of both the public and private of data and information. The AMU Cloud-Net prototype was designed and demonstrated over premium version of Interact Intranet technology platform for 45 days access 


\section{Results Discussion and Model Designing}

This research study developed a migration strategy model for shifting the intranet from traditional on premise infrastructure to the cloud infrastructures. As presented in the fig. 2, this model is designed and proposed for next generations intranets where features like on-demand Scalability, Open boundary Accessibility, Service reliability, High Availability (Uptime), High responsive, Fault tolerant with promised Security, Intelligent Search Content Filtration, Cost-effectiveness, and Anytime, Anywhere over Any device Accessibility can be incorporated or enhanced and named as "DPS-AA Migration Strategy Model". Before designing the migration model, the study conducted survey, interview and technical observation for primary data collection and analysis. The technical staff (technicians), experts (director, network admin, and system admin), end users (academic staff \& students) and managing authorities in the University (vice presidents, directors and HODs) participated in survey and interview. They provided in-depth inputs and the facts about issues, limitations and challenges of the existing state of art intranet in the university system. From their responses, it was revealed that $97.37 \%$ respondents are aware about cloud and $89.47 \%$ are using cloud services with promised trust and benefits and interested to have an alternative model/solution for Intranet for modernizing and upgrading the intranet features or services with enhanced performance of the existing Intranet.

The fig. 2 presents the DPS-AA Migration Strategy Model after investigation and analysis of the primary data towards cloud technology and services for the proposed intranets. In the functional demonstration, the respondents were asked to select only one service from the given services over the prototype designed for functional of Corporate Intranet like boundary-less accessibility, all-time availability with $99.99 \%$ uptime and others. The proposed migration strategy model based intranet over cloud shown the promising features which are not available in the existing state of art on premise intranet over local servers. The major contribution of this research is to show an alternative pathway from migration of the old fashioned intranets running over the on premise servers to the cloud. During the functional demo and survey it was clearly revealed that the current state of art intranets in the educational institutions of Ethiopia are not scalable, cost effective, rapidly upgradable, reliable, all time available with zero down time, and intelligent, and boundary-less accessible. In in order to alleviate the aforementioned issues and challenges, the proposed migration strategy model can be a better solution for the future generation of Intranets in Ethiopia.

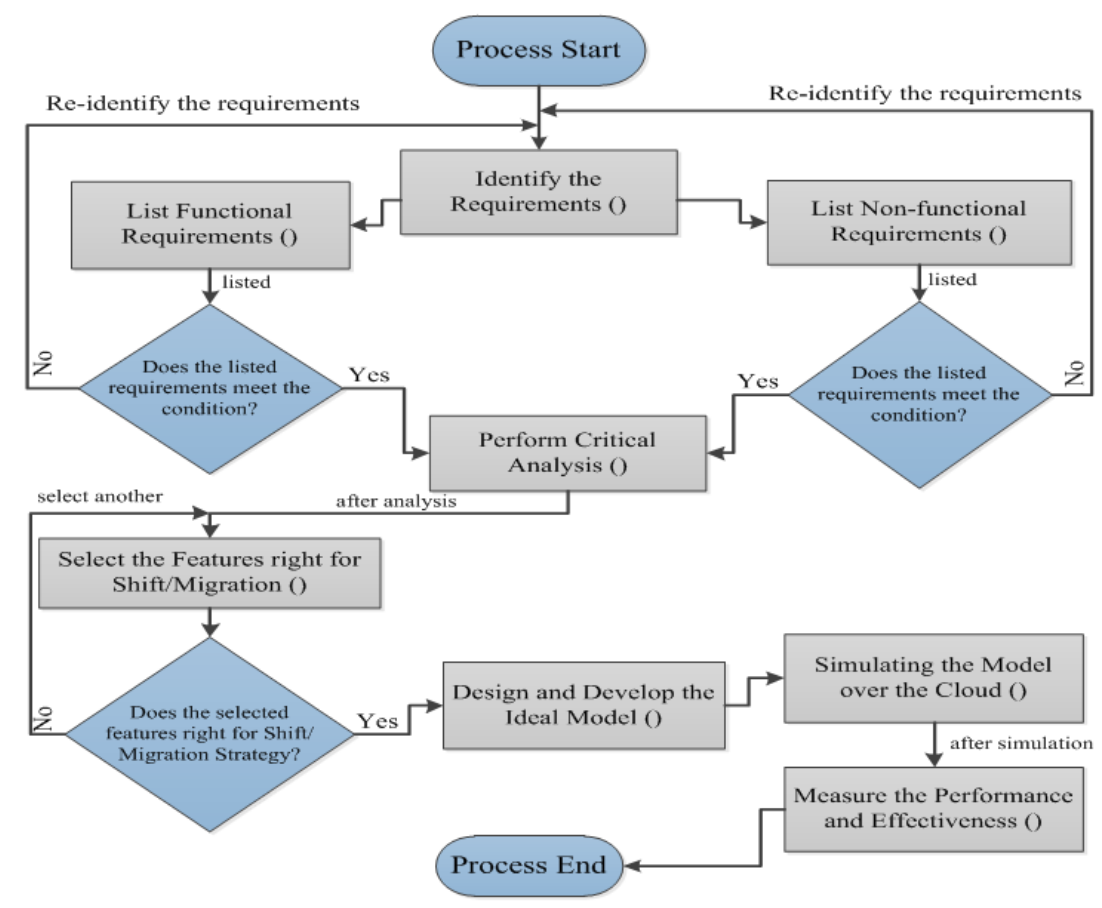

Fig. 2. DPS-AA Migration Strategy Model (Cloud-Net)

As presented in Fig. 2, the migration strategy model i.e. DPS-AA was designed as roadmaps to consider different issues before strategic migration of the intranet over cloud. From the flow of the model, it was observed that the migration strategy needs to identify the two most important needs, 1) functional and 2) non-functional requirements to provide decision support for migrating on-premise intranets over the cloud. The description for migration strategy model is as follows: 
i. Identification \& Analysis of Functional Requirements: The functional requirements that can be identified for the smooth migration of the intranet over cloud in the proposed study are -

1) Identifying and analyzing data storages needs and data services before Migration/Migration process.

2) Gathering technical details (re-design issues) of On-premise and Off-premise support before Migration process.

3). Analyzing the Management and Configuration capabilities for data storages and data services before Migration process,

4) Identification of application compatibilities and resolution (i.e. whether the migrated intranet will be supported by different data services over the cloud as expected or not?)

ii. Identification \& Analysis of Non-functional Requirements: In addition to the functional requirements, potential nonfunctional requirements are also proposed to be investigated and analyzed for a smooth migration of the intranet over cloud. The non-functional requirements that bring significant benefits to the organization as a general and end user's community as a special are required to be analyzed. These are: -

1) Data Security and Privacy,

2) Infrastructure \& Platform Scalability,

3) Open Boundary Accessibility,

4) High Service Availability,

5) Cost-Effectiveness, and

6) Additional Add-On features, which are not present in the existing state of art Models (like Notification for Academic and Management Staff Leaders, Intelligent Search/Content Filtration Features, and User's \& Application level Categorization).

iii. Feature Based Analysis of the On-Premise vs. Cloud based Intranet: At this phase, data and facts are required to be collected in the above mentioned phases (i.e. functional and non-functional requirements) for comparative analysis and documentation.

iv. Selection of frequently used features that meet the objective of the proposed Strategy: In this phase, some of the extended add-on features, which are mostly required and popularly desired and accepted by user's community of the organization, are required to be selected for the proposed strategic model. These may be like search feature, project management and collaboration etc.

v. Designing and Developing the Conceptual Intranet Migration Strategy Model over Cloud: In this phase, enhanced performance measures, add-on features which are mostly selected and popularly accepted by user's communities are required to be considered for conceptual design of the Ideal Intranet over Cloud Platforms.

vi. Functional Demonstration of Intranet over Cloud: At this phase, an Ideal Intranet Prototype is required to be designed, developed and demonstrated over the selected Cloud Service Providers Infrastructures.

vii. Measuring the performance and effectiveness of the prototype of the Intranet over Cloud: Finally, performance of the Intranet migration Strategy model over Cloud can be measured for its performance, features and efficiency.

\section{Conclusion}

This research study is an effort to explore the new knowledge contribution in constructing a migration strategy model for Intranets over Cloud. The study was focused on how different organization's traditional computing platforms and intranets are challenged in terms of poor performance and lack of advanced features. The research considered case based analysis of the AMU's Intranet. The research study tried to investigate and analyze the issues, challenges and limitations in existing state of art intranets and their performance, features by analyzing the satisfaction levels of the participants. Finally, the research designed a Migration Strategy Model named as "DPS-AA Model" for the Intranet over Cloud Platforms towards enhancing the performance and extending the features like on-demand Scalability, Open boundary Accessibility, Service reliability, High Availability (Uptime), High responsive, Fault tolerant with promised Security, Intelligent Search Content Filtration, Cost-effectiveness, and Anytime, Anywhere over Any device Accessibility.

As a contextual analysis of salient features of the intranet networks; this research conducted feature based analysis of three different types of computer networks namely Internet (the world-wide network of computers accessible to anyone), Intranet (a network that is not available to the world outside of the Intranet), and Extranet (an Intranet that is partially accessible to authorized outsiders). According to a generalized fact, cloud computing is a next generation computing paradigm, which uses a Computer Network (i.e. Internet) as a network backbone for delivering computing, communication and collaboration services. In order to develop a Migration Strategy Model for Intranet over Cloud Platform in general and AMU Intranet over Cloud i.e. AMU Cloud-Net as a special case, this research used different fact finding techniques for gathering qualitative and quantitative data. Finally, a Migration Strategy Model (DPS-AA) for Intranet over Cloud in general and AMU Cloud-Net using Interact Intranet tool as a special case was designed and 
demonstrated for the performance evaluation. The designed migration strategy model and its functional prototype with limited features have shown the promised results towards performance enhancements and Add-On features in the existing state of art intranets. These result oriented conclusions showcased new pathways towards strong needs for modernization of intranets over clouds.

\section{Recommendations}

In this research study, the researchers tried to cover several factors to meet the objectives of this study. It is difficult to carry out multiple tasks in a defined scope of the research and time given to achieve the research goal. The following lists of recommendations are forwarded for further studies:

1. In this study, the Intranet prototype over cloud was designed, developed and evaluated using free trial version tools and technologies only. Therefore, it's recommended for the future researchers to use commercial version of the Intranet development tool (i.e. the tool with full features and functionalities)

2. Secondly, the Intranet i.e. AMU Cloud-Net is modeled over Cloud Platforms for Educational Institutions in general and Arba Minch University Intranet as a specific case. It is quite possible that all educational institutions may not have the same structure, working culture and work preferences and therefore, the Migration Model may be adopted with smart contextualization and customization.

3. Again the researcher recommends that designing and developing a fully-featured Intranet migration model for integrating all Ethiopian Educational Institutions towards Sharing of experiences, Computing, Communication and Collaborating Resources can be an another fold of new knowledge research.

\section{References}

[1] Convergence of Intranetware in Project Management for Effective Enterprise Management. Sharma, Durga Prasad. 2, 2009, Journal of Global Information Technology (JGIT)-USA, Vol. 4, pp. 65-85.

[2] Development of Intranet Application on Oracle Cloud. Bose, Saumendu and Kumar, Saurabh. 5, May 2014, International Journal of Emerging Technology and Advanced Engineering, Vol. 4, pp. 430-434.

[3] Migrating Enterprise Applications to the Cloud: Methodology and Evaluation. Strauch, Steve, et al., et al. 2, LeinfeldenEchterdingen, Germany : \{Perpetual Innovation Media Pvt. Ltd, 2014, International Journal of Big Data Intelligence, Vol. 1, pp. 127-140.

[4] Yadav, Dharmveer, Sharma, D P And Keswani, Bright. Porting Intranet Over Cloud For Educational Service Amplification (Special Reference To Higher Educational Institutions). 2017. Porting Intranet Over Cloud For Educational Service Amplification.

[5] Pahl, Claus, Xiong, Huanhuan and Walshe, Ray. A Comparision of On-premise to Cloud Migration Approaches. Dublin City University : s.n.

[6] Team, Corporate Editorial. Migrating Applications to Public Cloud Services Roadmap for Success. s.1. : Cloud Standards Customer Counsil, 2013.

[7] Cloud Migration Architecture and Pricing - Mapping a Licensing Bussiness Model for Software Vendors to a SaaS Bussiness Model. Fowley, Frank and Pahl, Claus. Dublin City University : s.n., 2016.

[8] Methods: Migration from On-premise to Cloud. Sabiri, Khadija and Benabbou, Faouzia. 2, 2015, IOSR Journal of Computer Engineering, Vol. 17, pp. 58-65.

[9] Corporate Editors. Work Smarter, Anywhere, with Hosted Email for Business. [Online] Microsoft Corporation, 2017. [Cited: January 10, 2017.] https://products.office.com/en-us/exchange/exchange-online.

[10] Joch, Alan. When Should Enterprises Replace Servers? BizTech. [Online] JUNE $14,2012$. http://www.biztechmagazine.com/article/2012/06/when-should-enterprises-replace-servers.

[11] Virtualization in Cloud Computing. L, Malhotra, D, Agarwal and A, Jaiswal. 2014, Journal of Information Technology \& Software Engineering. 2165- 7866.

[12] Vivek Thakur ; Tim Eisenhauer. Intranet and Social Collaboration Solutions. [Online] Axero Solutions LLC, 2007. [Cited: 01 10, 2017.] https://axerosolutions.com/.

[13] Corporate Editorial Groups. Interact Intranet Cloud. https://www.interactintranet.com.au. [Online] [Cited: January 23, 2017.] https://www.interactintranet.com.au/interact-intranet-cloud/.

[14] Briggs, Barry and Kassner, Eduardo. Enterprise Cloud Strategy. [ed.] Karen Szall, Dianne Russell and Bob Russell. Washington : Microsoft Press, 2016. 978-1-5093-0196-6.

[15] Educational Institution Intranet Zone: Scalability to Cloud. (Ph.D.), Nidhi Srivastava and (Ph.D.), Rajiv Pandey. 5, 2016, International Journal of Computer Applications (0975 - 8887), Vol. 133, p. January.

[16] Development of Intranet App with JAVA on Oracle Cloud. Bose, Saumendu and Kumar, Saurabh. 5, May 2014, International Journal of Emerging Technology and Advanced Engineering, Vol. 4, pp. 430-434.

[17] Boothby, Alison. Building a Modern intranet: A Communicator's Guide to Collaborative Platforms. s.l. : IGLOO, 2016.

[18] A Comparative Study of Different Deployment Models in a Cloud. Parsi, Kalphana and Laharika, M. 5, Hyderabad, India : s.n., May 2013, International Journal of Advanced Research in Computer Science and Software Engineering, Vol. 3, pp. 512-515.

[19] Cloud Computing Security through Cryptography for Banking Sector. Shekhawat, Narpat Singh and Sharma, Durga Prasad. New Delhi : s.n., 2011. Computing For National Development. 978-93-80544-00-7. 
[20] A Study of Intranet over Cloud. Yadav, Dharmveer, Sharma, Prof.(Dr.) D P and Keshwani, Dr. Bright. 2, March 2017, International Journal of New Innovations in Engineering and Technology, Vol. 7. 2319-6319.

[21] Cloud Computing Security through Cryptography for Banking Sector. Shekhawat, Narpat Singh and Sharma, Durga Prasad. New Delhi : INDIACom-2011, 2011. Computing For National Development. 978-93-80544-00-7.

[22] Lundqvist, Andreas, Johansson, Niklas and Yildiz, Simon. Intranet for Project and Document Management. 2013.

[23] Hybrid cloud computing in e-governance: Related security risks and solutions. Sharma, DP and Shekhawat, Hardayal Singh. 1, s.l. : Maxwell Science Publishing, 2012, Research Journal of Information Technology, Vol. 4, pp. 1-6.

[24] Suitability Analytics and Cloud Computing Adoption Modeling for Educational Institutions. Sharma, Dr. DP and Alemseged, Kassahun. 3, July-September 2016, International Journal of Information Technology \& Computer Sciences Perspectives, Vol. 5.

\section{Authors' Profiles}

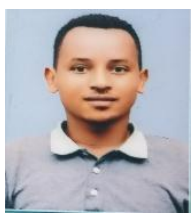

Abebe Alambo is a lecturer of Computer Science Department in Gambella University, Ethiopia. He earned his Bachelor and Master's degree in Computer Science from Arba Minch University, Ethiopia. Along with teaching learning process, he is participating in research and community services as well as he delivers short term training in computing and IT domain for administrative staffs and students within the campus and outside the campus. He is also committed in research and project works. He is interested in Cloud Computing and Big Data Analytics research fields.

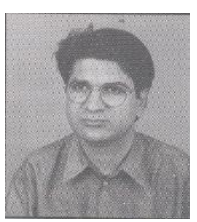

Durga Prasad Sharma is a Professor and Research Adviser. Apart from his scholarly scientific services to the salient universities of the developing and developed countries like AMUiT Ethiopia, RTU India, VUB Belgium and UPS, France, he holds MS \& PhD Degrees in Computing and Intranetwares. For more details about him http://dpsharma.info and other web world sources can be accessed.

How to cite this paper: Abebe Alambo Tona, Durga Prasad Sharma, " DPS-AA: Intranet Migration Strategy Model for Clouds", International Journal of Modern Education and Computer Science(IJMECS), Vol.12, No.5, pp. 55-63, 2020.DOI: 10.5815/ijmecs.2020.05.05 